\title{
First Measurement of the Charge Asymmetry in Beauty-Quark Pair Production
}

\author{
R. Aaij et al. \\ (LHCb Collaboration)
}

(Received 19 June 2014; published 20 August 2014)

\begin{abstract}
The difference in the angular distributions between beauty quarks and antiquarks, referred to as the charge asymmetry, is measured for the first time in $b \bar{b}$ pair production at a hadron collider. The data used correspond to an integrated luminosity of $1.0 \mathrm{fb}^{-1}$ collected at $7 \mathrm{TeV}$ center-of-mass energy in protonproton collisions with the $\mathrm{LHCb}$ detector. The measurement is performed in three regions of the invariant mass of the $b \bar{b}$ system. The results obtained are $A_{\mathrm{C}}^{b \bar{b}}\left(40<M_{b \bar{b}}<75 \mathrm{GeV} / c^{2}\right)=0.4 \pm 0.4 \pm 0.3 \%$, $A_{\mathrm{C}}^{b \bar{b}}\left(75<M_{b \bar{b}}<105 \mathrm{GeV} / c^{2}\right)=2.0 \pm 0.9 \pm 0.6 \%, \quad A_{\mathrm{C}}^{b \bar{b}}\left(M_{b \bar{b}}>105 \mathrm{GeV} / c^{2}\right)=1.6 \pm 1.7 \pm 0.6 \%$, where $A_{\mathrm{C}}^{b \bar{b}}$ is defined as the asymmetry in the difference in rapidity between jets formed from the beauty quark and antiquark, where in each case the first uncertainty is statistical and the second systematic. The beauty jets are required to satisfy $2<\eta<4, E_{\mathrm{T}}>20 \mathrm{GeV}$, and have an opening angle in the transverse plane $\Delta \phi>2.6 \mathrm{rad}$. These measurements are consistent with the predictions of the standard model.
\end{abstract}

Measurements in $p \bar{p}$ collisions at the Tevatron [1-6] suggest that (anti)top quarks are produced along the (anti) proton beam direction more often than predicted by the standard model (SM) [7]. Many extensions to the SM have been proposed to explain this discrepancy (for a review, see Ref. [8]) that couple new particles to quarks in a variety of ways. Therefore, constraints on quark-antiquark production charge asymmetries other than top anti top $(t \bar{t})$ could discriminate between models and be used as a probe of non-SM physics. For example, some theories proposed to explain the Tevatron results also predict a large charge asymmetry in $b \bar{b}$ production $[9,10]$. No measurement has been made to date of the $b \bar{b}$ charge asymmetry at a hadron collider.

The symmetric initial state of proton-proton collisions at the LHC does not permit a charge asymmetry to be manifest as an observable defined using the direction of one beam relative to the other. However, the asymmetry in the momentum fraction of quarks and antiquarks inside the proton means that a charge asymmetry can lead to a difference in the rapidity distributions of beauty quarks and antiquarks. The $b \bar{b}$ charge asymmetry in $p p$ collisions is defined as

$$
A_{\mathrm{C}}^{b \bar{b}} \equiv \frac{N(\Delta y>0)-N(\Delta y<0)}{N(\Delta y>0)+N(\Delta y<0)}
$$

where $\Delta y \equiv\left|y_{b}\right|-\left|y_{\bar{b}}\right|$ is the rapidity difference between jets formed from the $b$ and $\bar{b}$ quarks. Measurements of the

* Full author list given at the end of the article.

Published by the American Physical Society under the terms of the Creative Commons Attribution 3.0 License. Further distribution of this work must maintain attribution to the author(s) and the published articles title, journal citation, and DOI. top-quark charge asymmetry by the ATLAS and CMS experiments are consistent with the SM expectations [11-13]. However, the large $g g \rightarrow t \bar{t}$ cross section at the LHC dilutes the observable signal of new physics entering the $q \bar{q} \rightarrow t \bar{t}$ process that dominates $t \bar{t}$ production at the Tevatron.

In the SM, the only sizable leading-order (LO) contribution to $A_{\mathrm{C}}^{b \bar{b}}$ comes from $Z \rightarrow b \bar{b}$ decays. The contribution of $Z \rightarrow b \bar{b}$ to $A_{\mathrm{C}}^{b \bar{b}}$ in a region of invariant mass of the $b \bar{b}$ system $\left(M_{b \bar{b}}\right)$ around the $Z$ boson mass is expected to be about $2 \%$ based on simulation. Production of $b \bar{b}$ pairs at LO in quantum chromodynamics (QCD) is symmetric under the exchange of $b$ and $\bar{b}$ quarks. At higher orders, radiative corrections to the $q \bar{q} \rightarrow b \bar{b}$ process generate an asymmetry in the differential distributions of the $b$ and $\bar{b}$ quarks and induce a correlation between the direction of the $b(\bar{b})$ quark and that of the incoming $q(\bar{q})$ quark. Such higher-order corrections are expected to be negligible at low $M_{b \bar{b}}$ and to increase in importance at larger $M_{b \bar{b}}$. The contribution to $A_{\mathrm{C}}^{b \bar{b}}$ from higher-order terms is expected to reach $1 \%$ near the $Z$ boson mass [14]. Precision measurements of $A_{\mathrm{C}}^{b \bar{b}}$ as a function of $M_{b \bar{b}}$ are sensitive probes of physics beyond the SM.

This Letter reports the first measurement of the charge asymmetry in beauty-quark pair production at a hadron collider. The data used correspond to an integrated luminosity of $1.0 \mathrm{fb}^{-1}$ collected at $7 \mathrm{TeV}$ center-of-mass energy in $p p$ collisions with the LHCb detector. The measurement is performed in three regions of $M_{b \bar{b}}: 40<$ $M_{b \bar{b}}<75 \mathrm{GeV} / c^{2}, \quad 75<M_{b \bar{b}}<105 \mathrm{GeV} / c^{2}, \quad$ and $M_{b \bar{b}}>105 \mathrm{GeV} / c^{2}$. This scheme is chosen such that the middle region is centered around the mass of the $Z$ boson and contains most of the $Z \rightarrow b \bar{b}$ candidates. The measurement is corrected to a pair of particle-level jets, each with a pseudorapidity $2<\eta<4$, transverse energy 
$E_{\mathrm{T}}>20 \mathrm{GeV}$, and an opening angle between the jets in the transverse plane $\Delta \phi>2.6 \mathrm{rad}$.

The $\mathrm{LHCb}$ detector is a single-arm forward spectrometer covering the range $2<\eta<5$ designed for the study of particles containing $b$ or $c$ quarks, described in detail in Refs. [15-18]. The trigger [19] consists of a hardware stage, based on information from the calorimeter and muon systems, followed by a software stage, which applies a full event reconstruction. Identification of beauty-hadron decays in the software trigger requires a two-, three-, or four-track secondary vertex with a large sum of the transverse momentum $\left(p_{\mathrm{T}}\right)$ of the tracks and a significant displacement from the primary $p p$ interaction vertices. A multivariate algorithm [20] is used for the identification of vertices consistent with the decay of a beauty hadron. This so-called topological trigger algorithm (TOPO) is also used in this analysis to identify the hadrons that contain the beauty quark and antiquark in $b \bar{b}$ pair production. The charge of the beauty (anti)quarks is determined by the charge of muons originating from semileptonic beauty-hadron decays.

Simulated events are used to calibrate the jet energy scale, to determine the reconstruction and selection efficiencies, and to unfold the detector response. In the simulation, $p p$ collisions are generated using PYTHIA [21] with a specific LHCb configuration [22]. Decays of hadronic particles are described by EVTGEN [23], in which final state radiation is generated using Рнотоs [24]. The interaction of particles with the detector and its response are implemented using the GEANT4 toolkit [25] as described in Ref. [26].

The $b \bar{b}$ are reconstructed as jets using the anti- $k_{\mathrm{T}}$ algorithm [27] with distance parameter $R=0.7$, as implemented in FASTJET [28]. The inputs to the jet reconstruction are selected using a particle flow approach [29]. Information from all the detector subsystems is used to create charged and neutral particle inputs to the jet algorithm. Jet-quality criteria are applied to remove jets for which a large fraction of the energy is likely due to sources other than a $p p$ collision, e.g., detector noise or poorly reconstructed tracks. The per jet efficiency of these criteria is $90-95 \%$ depending on the jet kinematic properties. The mean number of $p p$ collisions per event is only 1.8 , making it unlikely to produce $b \bar{b}$ in separate collisions; however, to prevent this, both jets are required to originate from the same $p p$ collision.

The observed energy of each jet is corrected to the particle-level energy accounting for the following effects: imperfect detector response; the presence of detector noise; energy contributions from $p p$ interactions other than the one in which the $b \bar{b}$ are produced; beauty (anti)quark energy flowing out of the jet cone; and the presence of a neutrino from the semileptonic decay of a beauty hadron in the jet. The jet energy correction varies in the range $0-20 \%( \pm 10 \%)$ for jets that do(do not) contain a neutrino from a semileptonic beauty-hadron decay. The mean value for jets that do not contain a semileptonic-decay neutrino is about $1 \%$. This correction is obtained from simulation and depends on the jet $\eta, E_{\mathrm{T}}$, and the number of $p p$ interactions in the event. Only jets in a well-understood kinematic regime of $\mathrm{LHCb}, E_{\mathrm{T}}>20 \mathrm{GeV}$ and $2<\eta<4$, are considered in this analysis. The relative resolution on $M_{b \bar{b}}$ obtained using these jets is about $15 \%$.

Jets in events selected by the TOPO need to be identified (tagged) as containing a beauty quark or antiquark (bTAG). For this task, an association is made between jets and the multitrack TOPO objects. If at least $60 \%$ of the detector hits that make up the tracks forming the TOPO object also belong to tracks within the jet, then the jet satisfies a bTAG requirement. At least one jet in the event is required to contain a beauty-hadron decay selected by the TOPO which caused the event to be recorded. The TOPO is applied to off-line-reconstructed tracks with a looser requirement to search for a second beauty-hadron decay in the event. If such a decay is found, and if it can be associated to another jet, then the event is identified as containing a $b \bar{b}$ pair. The mean di-bTAG efficiency for dijet events used in this analysis is about $30 \%$, while the per jet mistag efficiency for jets initiated by light quarks and gluons is less than $0.1 \%$. To enhance the contribution of non-gg production mechanisms, $\Delta \phi>2.6 \mathrm{rad}$ is required between the two jets that satisfy the bTAG requirement.

The largest background contribution is due to charm jets. The level of background contamination is determined using the so-called corrected mass

$$
M_{\text {corr }}=\sqrt{M^{2}+\left(\frac{p}{c}\right)^{2} \sin ^{2} \theta}+\frac{p}{c} \sin \theta,
$$

where $M$ and $p$ are the invariant mass and momentum of all tracks in the jet that are inconsistent with originating directly from a $p p$ collision and have a minimum distance of closest approach to a track used in the TOPO less than $0.2 \mathrm{~mm}$. The angle $\theta$ is between the momentum and the direction from the $p p$ collision to the TOPO object vertex. The corrected mass is the minimum mass the long-lived hadron can have that is consistent with the direction of flight.

Figure 1 shows the corrected-mass distribution. The corrected-mass probability density functions (PDFs) for beauty and charm are obtained from simulation. Imperfect measurement of the direction of flight can result in a larger corrected mass than the true hadron mass. For charmhadron decays, the particles originate from a single point in space and typically the missing momentum is carried by a single low-mass particle, thus, the corrected mass peaks near the known charm-meson mass. The vast majority of beauty-hadron decays involve intermediate charm hadrons which results in not all stable particles originating from the same spatial point. The missing momentum is typically 
carried away by multiple particles and the invariant mass of the missing momentum may be large. Hence, the corrected mass for beauty decays peaks below the known beautymeson mass and has worse resolution than for charm. The result of a fit to the data shown in Fig. 1 is that $3.6 \pm 1.2 \%$ of events in the final sample are not $b \bar{b}$, where the uncertainty is due to the corrected-mass PDFs. The contribution from jets initiated by light quarks or gluons is found to be negligible. Furthermore, the limited acceptance of the $\mathrm{LHCb}$ detector for $b \bar{b}$ originating from $t \bar{t}$ makes this contribution negligible as well.

To measure the charge asymmetry, the charge of the beauty (anti)quark needs to be identified in at least one of the jets (qTAG). The qTAG requirement is that a track in the TOPO object and in the jet is identified as a muon. The muon is required to satisfy $p_{\mathrm{T}}>2 \mathrm{GeV} / c$ and $p>$ $10 \mathrm{GeV} / c$ to reduce the charge asymmetry due to detector biases. This strategy is designed to look for muons coming from semileptonic beauty-hadron decays; thus, the charge of the muon tags the charge of the beauty quark or
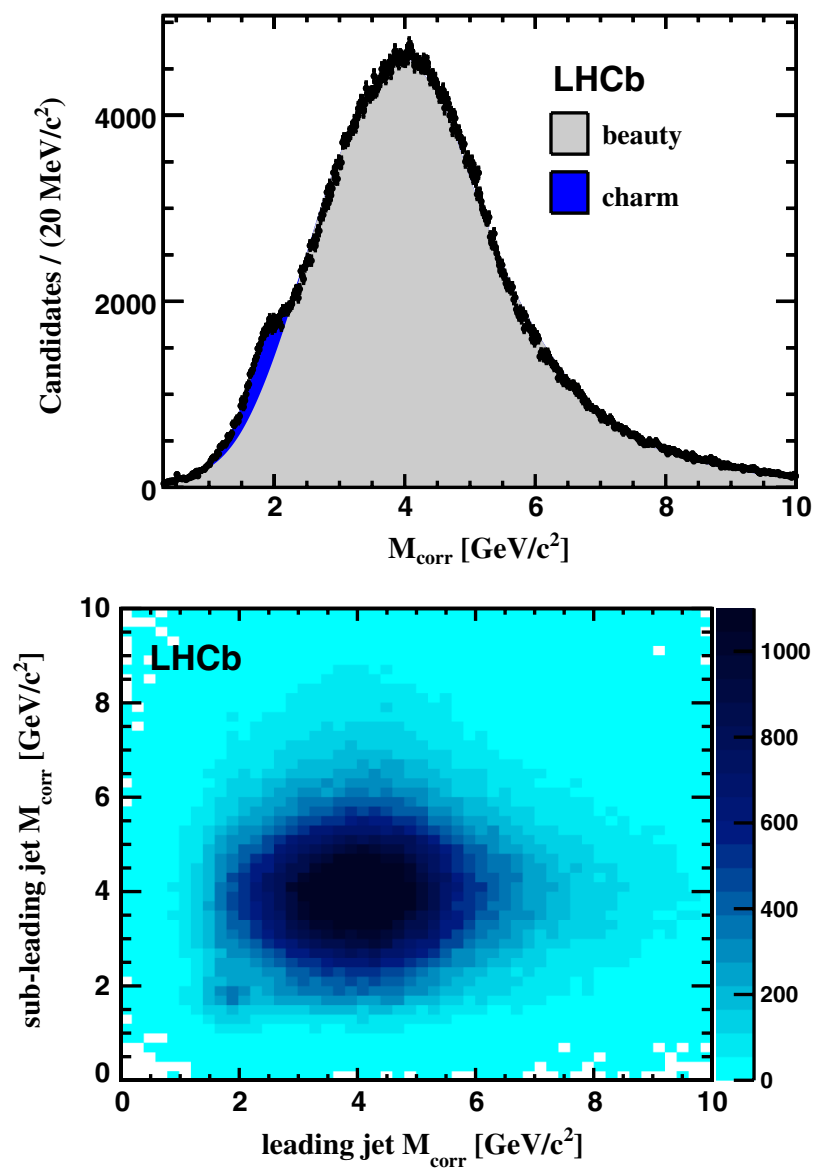

FIG. 1 (color online). (top) Corrected mass of TOPO objects associated to bTAG jets in the final event sample. Less than $2 \%$ of jets are found to originate from charm. (bottom) Corrected mass of TOPO objects associated to subleading vs. leading jets in the final event sample. A small $c \bar{c}$ contribution is visible near $(2,2) \mathrm{GeV} / c^{2}$. antiquark. Decays of the type $b \rightarrow c \rightarrow \mu$ contaminate the charge tagging. To mitigate this, the tagging muon is required to have the highest momentum of all displaced tracks in the jet. A further dilution to the charge-tagging purity arises due to oscillations of the $B^{0}$ and $B_{s}^{0}$ mesons.

The expected qTAG purity, defined as the probability to correctly assign the charge of the beauty quark in a qTAG jet, can be estimated using the following: the measured $b$-hadron production fractions [30,31]; the $b$-hadron and $c$-hadron semileptonic branching fractions [32]; the chargetagging efficiencies for $b$-and $c$-hadron semileptonic decays obtained from simulation; the $B^{0}$ and $B_{s}^{0}$ oscillation frequencies $[33,34]$ and the reconstruction efficiency as a function of $b$-hadron lifetime obtained from simulation. Combining all of this information yields an expected qTAG purity of $73 \pm 4 \%$. The purity is expected to decrease by a few percent with increasing jet energy due to an increase in the neutral-beauty-meson production fractions.

The qTAG purity is measured directly using events where both bTAG jets also satisfy the qTAG requirement using the fraction of events where the two muons have opposite charges. This gives an integrated qTAG purity of $70.3 \pm 0.3 \%$, which agrees with the predicted value, and values of $71.6 \pm 0.5 \%, 68.8 \pm 0.8 \%$, and $66.1 \pm 1.9 \%$ for $40<M_{b \bar{b}}<75 \mathrm{GeV} / c^{2}, 75<M_{b \bar{b}}<105 \mathrm{GeV} / c^{2}$, and $M_{b \bar{b}}>105 \mathrm{GeV} / c^{2}$, respectively. The observed decrease in purity agrees with expectations. The qTAG purity is found to be consistent in data for all $\Delta y$. As a further consistency check, a separate study of the qTAG purity is performed using events with a jet and a fully reconstructed self-tagging $B^{+} \rightarrow J / \psi K^{+}$or $B^{+} \rightarrow \bar{D}^{0} \pi^{+}$decay. In these events, the charge of the $B^{+}$provides an unambiguous qTAG of the beauty jet for $b \bar{b}$ pair production. Using $B^{+}+$ jet events where the jet satisfies the qTAG, the qTAG purity is determined to be $73 \pm 3 \%$. This result agrees with both

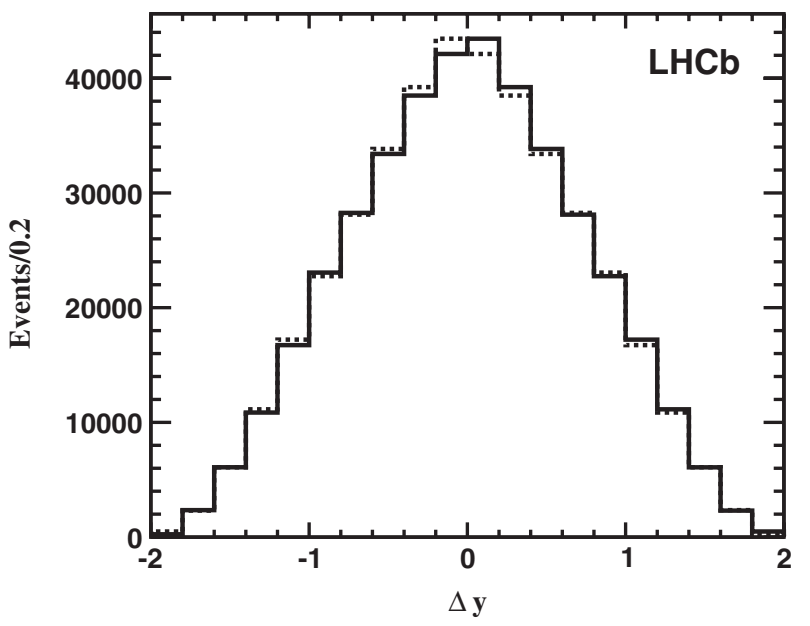

FIG. 2. Reconstructed $\Delta y$ distribution for all selected events after background subtraction and correction for qTAG impurity. The dashed line shows the distribution reflected about the vertical axis. 


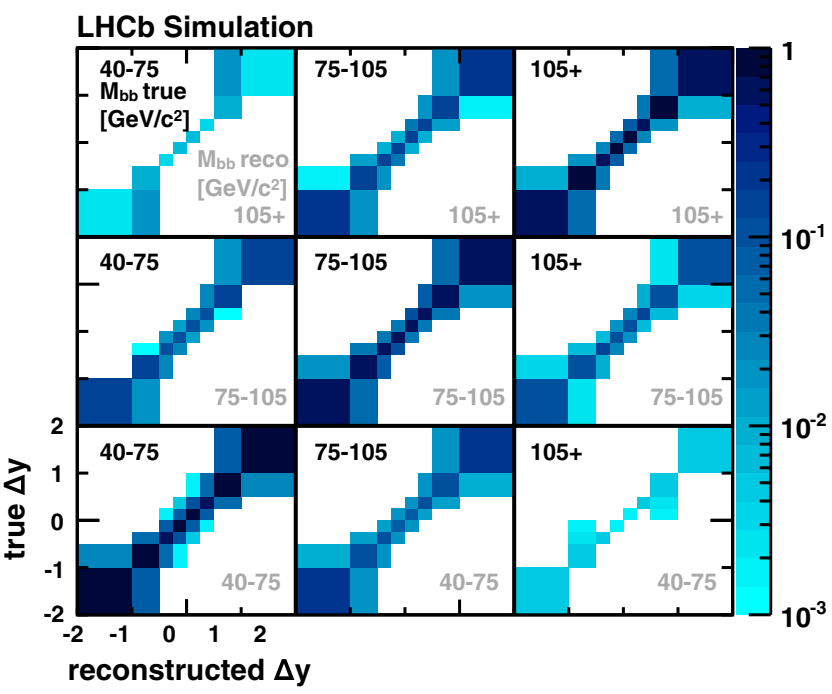

FIG. 3 (color online). Migration matrix in $\Delta y$ and $M_{b \bar{b}}$.

the predicted and di-qTAG results. The di-qTag purity measurement is used to obtain the final $A_{\mathrm{C}}^{b \bar{b}}$ results below.

Figure 2 shows the $\Delta y$ distribution after background subtraction and correcting for qTAG impurity. The reconstructed distributions of $\Delta y$ and $M_{b \bar{b}}$ are corrected for the effects of detector resolution and for event reconstruction and selection efficiency. The correction for detector resolution is achieved by applying a two-dimensional unfolding procedure to the data [35]. The migration matrix in $\Delta y$ and $M_{b \bar{b}}$ is shown in Fig. 3. The selection efficiency is obtained from simulated events as a function of $\Delta y$ and $M_{b \bar{b}}$. The residual dependence of the efficiency on other jet kinematic variables has a negligible impact on the resulting measurement of $A_{\mathrm{C}}^{b \bar{b}}$.

The main sources of systematic uncertainties on the measurement of $A_{\mathrm{C}}^{b \bar{b}}$ are as follows: precision of the qTAG purity and its dependence on jet kinematic properties; uncertainty in the unfolding; determination of the selection efficiency; and any residual detector-related asymmetries. Table I summarizes the values of the systematic uncertainties assigned to the measurement of $A_{\mathrm{C}}^{b \bar{b}}$ in each $M_{b \bar{b}}$ region. Measurement of the qTAG purity is data driven and the statistical uncertainties are propagated to $A_{\mathrm{C}}^{b \bar{b}}$ to determine the systematic uncertainty. The uncertainty due to unfolding accounts for the choice of data sample used to generate the migration matrix and mismodeling of the detector response in the simulation. The uncertainty due to efficiency is dominated by the statistical uncertainty of the simulation. The polarity of the LHCb dipole magnet is reversed periodically. This coupled with the hard momentum spectrum of the tagging muons results in only small detection-based asymmetries. Additionally, due to the definition of $\Delta y$, these detection asymmetries cancel to very good approximation when summing over $\mu^{+}$and $\mu^{-}$ tags. The detection asymmetry of charged kaons causes a negligible bias in $A_{\mathrm{C}}^{b \bar{b}}$.
TABLE I. Absolute systematic uncertainties.

\begin{tabular}{lccc}
\hline \hline & \multicolumn{3}{c}{$M_{b \bar{b}}\left(\mathrm{GeV} / c^{2}\right)$} \\
\hline Source & $(40,75)$ & $(75,105)$ & $>105$ \\
Mis-qTAG & $\ldots$ & $0.1 \%$ & $0.2 \%$ \\
Unfolding & $0.3 \%$ & $0.6 \%$ & $0.4 \%$ \\
$\varepsilon\left(M_{b \bar{b}}, \Delta y\right)$ & $0.1 \%$ & $0.1 \%$ & $0.2 \%$ \\
$\varepsilon(b)-\varepsilon(\bar{b})$ & $\ldots$ & $\ldots$ & $0.3 \%$ \\
Total & $0.3 \%$ & $0.6 \%$ & $0.6 \%$ \\
\hline \hline
\end{tabular}

Figure 4 shows the corrected $\Delta y$ distribution summed over all $M_{b \bar{b}}$ regions considered $\left(M_{b \bar{b}}>40 \mathrm{GeV} / c^{2}\right)$. The LO SM prediction, which includes LO QCD and $Z \rightarrow b \bar{b}$, obtained from Pythia [36,37] is also shown. The SM uncertainty includes contributions from the renormalization and factorization scales, and from the parton distribution functions. A next-to-LO SM calculation is required to obtain $A_{\mathrm{C}}^{b \bar{b}}$ at the percent level. However, the LO result is sufficient to demonstrate agreement between the theory and unfolded $b \bar{b}$ pair-production distribution.

The measurement of $A_{\mathrm{C}}^{b \bar{b}}$ is performed in three regions of $M_{b \bar{b}}$ and the results obtained are

$$
\begin{aligned}
A_{\mathrm{C}}^{b \bar{b}}(40,75) & =0.4 \pm 0.4(\text { stat }) \pm 0.3(\text { syst }) \%, \\
A_{\mathrm{C}}^{b \bar{b}}(75,105) & =2.0 \pm 0.9(\text { stat }) \pm 0.6(\text { syst }) \%, \\
A_{\mathrm{C}}^{b \bar{b}}(>105) & =1.6 \pm 1.7(\text { stat }) \pm 0.6(\text { syst }) \%,
\end{aligned}
$$

where the ranges denote the regions of $M_{b \bar{b}}$ in units of $\mathrm{GeV} / c^{2}$. These measurements are the first to date of the charge asymmetry in $b \bar{b}$ pair production at a hadron collider. The results are corrected to a pair of particle-level jets each with $2<\eta<4, E_{\mathrm{T}}>20 \mathrm{GeV}$, and $\Delta \phi>2.6 \mathrm{rad}$

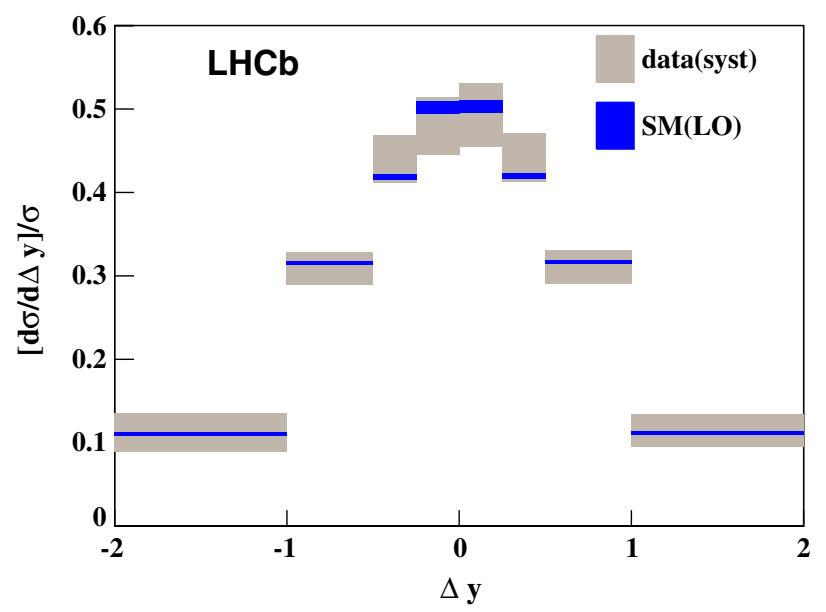

FIG. 4 (color online). Corrected $\Delta y$ distribution for all selected events. The statistical uncertainties are negligible. The systematic uncertainties are highly correlated from bin to bin and largely cancel in the determination of $A_{\mathrm{C}}^{b \bar{b}}$. The LO SM prediction obtained from PYTHIA [36,37] is also shown. 
between the jets. All results are consistent with the SM expectations.

We express our gratitude to our colleagues in the CERN accelerator departments for the excellent performance of the LHC. We thank the technical and administrative staff at the LHCb institutes. We acknowledge support from CERN and from the national agencies: CAPES, CNPq, FAPERJ, and FINEP (Brazil); NSFC (China); CNRS/IN2P3 (France); BMBF, DFG, HGF, and MPG (Germany); SFI (Ireland); INFN (Italy); FOM and NWO (Netherlands); MNiSW and NCN (Poland); MEN/IFA (Romania); MinES and FANO (Russia); MinECo (Spain); SNSF and SER (Switzerland); NASU (Ukraine); STFC (United Kingdom); NSF (USA). The Tier1 computing centers are supported by IN2P3 (France), KIT and BMBF (Germany), INFN (Italy), NWO and SURF (Netherlands), PIC (Spain), GridPP (United Kingdom). We are indebted to the communities behind the multiple open source software packages on which we depend. We are also thankful for the computing resources and the access to software $\mathrm{R} \& \mathrm{D}$ tools provided by Yandex LLC (Russia). Individual groups or members have received support from EPLANET, Marie SkłodowskaCurie Actions and ERC (European Union), Conseil général de Haute-Savoie, Labex ENIGMASS and OCEVU, Région Auvergne (France), RFBR (Russia), XuntaGal and GENCAT (Spain), Royal Society and Royal Commission for the Exhibition of 1851 (United Kingdom).

[1] V. M. Abazov et al. (D0 collaboration), Phys. Rev. D 84, 112005 (2011).

[2] V. M. Abazov et al. (D0 collaboration), Phys. Rev. D 88, 112002 (2013).

[3] V. M. Abazov et al. (D0 collaboration), arXiv:1403.1294.

[4] T. Aaltonen et al. (CDF collaboration), Phys. Rev. D 87, 092002 (2013).

[5] T. Aaltonen et al. (CDF collaboration), Phys. Rev. D 88, 072003 (2013).

[6] T. Aaltonen et al. (CDF collaboration), arXiv:1404.3698.

[7] W. Bernreuther and Z.-G. Si, Phys. Rev. D 86, 034026 (2012).

[8] J. Aguilar-Saavedra, D. Amidei, A. Juste, and M. PerezVictoria, arXiv:1406.1798.

[9] D. Kahawala, D. Krohn, and M. J. Strassler, J. High Energy Phys. 01 (2012) 069

[10] B. Grinstein and C. W. Murphy, Phys. Rev. Lett. 111, 062003 (2013).
[11] A. Chatrchyan et al. (CMS collaboration), J. High Energy Phys. 04 (2014) 191.

[12] A. Chatrchyan et al. (CMS collaboration), Phys. Lett. B 709, 28 (2012).

[13] G. Aad et al. (ATLAS collaboration), Eur. Phys. J. C 72, 2039 (2012).

[14] R. Gauld and U. Haisch (private communication).

[15] A. A. Alves Jr. et al. (LHCb collaboration), J. Instrum. 3, S08005 (2008).

[16] R. Arink et al., J. Instrum. 9, P01002 (2014).

[17] M. Adinolfi et al., Eur. Phys. J. C 73, 2431 (2013).

[18] A. A. Alves Jr. et al., J. Instrum. 8, P02022 (2013).

[19] R. Aaij et al., J. Instrum. 8, P04022 (2013).

[20] V. V. Gligorov and M. Williams, J. Instrum. 8, P02013 (2013).

[21] T. Sjöstrand, S. Mrenna, and P. Skands, J. High Energy Phys. 05 (2006) 026.

[22] I. Belyaev et al., in Nuclear Science Symposium Conference Record (NSS/MIC) (IEEE, New York, 2010), p. 1155.

[23] D. J. Lange, Nucl. Instrum. Methods Phys. Res., Sect. A 462, 152 (2001).

[24] P. Golonka and Z. Was, Eur. Phys. J. C 45, 97 (2006).

[25] J. Allison et al. (Geant4 collaboration), IEEE Trans. Nucl. Sci. 53, 270 (2006); S. Agostinelli et al. (Geant4 collaboration), Nucl. Instrum. Methods Phys. Res., Sect. A 506, 250 (2003).

[26] M. Clemencic, G. Corti, S. Easo, C. R. Jones, S. Miglioranzi, M. Pappagallo, and P. Robbe, J. Phys. Conf. Ser. 331, 032023 (2011).

[27] M. Cacciari, G. P. Salam, and G. Soyez, J. High Energy Phys. 04 (2008) 063.

[28] M. Cacciari and G. P. Salam, Phys. Lett. B 641, 57 (2006).

[29] R. Aaij et al. (LHCb collaboration), J. High Energy Phys. 01 (2014) 033.

[30] LHCb collaboration, Report No. LHCb-CONF-2013-011.

[31] R. Aaij et al. (LHCb collaboration), Phys. Rev. D 85, 032008 (2012).

[32] J. Beringer et al. (Particle Data Group), Phys. Rev. D 86, 010001 (2012), and 2013 partial update for the 2014 edition.

[33] R. Aaij et al. (LHCb collaboration), New J. Phys. 15, 053021 (2013).

[34] R. Aaij et al. (LHCb collaboration), Phys. Lett. B 719, 318 (2013).

[35] G. D’Agostini et al., Nucl. Instrum. Methods Phys. Res., Sect. A 362, 487 (1995).

[36] T. Sjöstrand, S. Mrenna, and P. Skands, Comput. Phys. Commun. 178, 852 (2008).

[37] J. Pumplin, D. R. Stump, J. Huston, H.-L. Lai, P. Nadolsky, and W.-K. Tung, J. High Energy Phys. 07 (2002) 012.

R. Aaij, ${ }^{41}$ B. Adeva, ${ }^{37}$ M. Adinolfi, ${ }^{46}$ A. Affolder,${ }^{52}$ Z. Ajaltouni, ${ }^{5}$ S. Akar, ${ }^{6}$ J. Albrecht,${ }^{9}$ F. Alessio, ${ }^{38}$ M. Alexander,${ }^{51}$ S. Ali, ${ }^{41}$ G. Alkhazov, ${ }^{30}$ P. Alvarez Cartelle, ${ }^{37}$ A. A. Alves Jr. ${ }^{25,38}$ S. Amato, ${ }^{2}$ S. Amerio, ${ }^{22}$ Y. Amhis, ${ }^{7}$ L. An, ${ }^{3}$ L. Anderlini, ${ }^{17, \mathrm{a}}$ J. Anderson, ${ }^{40}$ R. Andreassen, ${ }^{57}$ M. Andreotti, ${ }^{16, b}$ J. E. Andrews,${ }^{58}$ R. B. Appleby, ${ }^{54}$ O. Aquines Gutierrez, ${ }^{10}$ F. Archilli, ${ }^{38}$ A. Artamonov, ${ }^{35}$ M. Artuso, ${ }^{59}$ E. Aslanides, ${ }^{6}$ G. Auriemma, ${ }^{25, c}$ M. Baalouch, ${ }^{5}$ 
S. Bachmann, ${ }^{11}$ J. J. Back, ${ }^{48}$ A. Badalov ${ }^{36}$ V. Balagura, ${ }^{31}$ W. Baldini, ${ }^{16}$ R. J. Barlow ${ }^{54}$ C. Barschel,${ }^{38}$ S. Barsuk, W. Barter, ${ }^{47}$ V. Batozskaya,${ }^{28}$ V. Battista ${ }^{39}$ A. Bay,${ }^{39}$ L. Beaucourt, ${ }^{4}$ J. Beddow, ${ }^{51}$ F. Bedeschi, ${ }^{23}$ I. Bediaga,${ }^{1}$ S. Belogurov,${ }^{31}$

K. Belous, ${ }^{35}$ I. Belyaev, ${ }^{31}$ E. Ben-Haim, ${ }^{8}$ G. Bencivenni, ${ }^{18}$ S. Benson, ${ }^{38}$ J. Benton, ${ }^{46}$ A. Berezhnoy, ${ }^{32}$ R. Bernet,${ }^{40}$

M.-O. Bettler, ${ }^{47}$ M. van Beuzekom, ${ }^{41}$ A. Bien, ${ }^{11}$ S. Bifani, ${ }^{45}$ T. Bird, ${ }^{54}$ A. Bizzeti, ${ }^{17, d}$ P. M. Bjørnstad ${ }^{54}$ T. Blake, ${ }^{48}$ F. Blanc, ${ }^{39}$ J. Blouw,${ }^{10}$ S. Blusk,${ }^{59}$ V. Bocci, ${ }^{25}$ A. Bondar, ${ }^{34}$ N. Bondar, ${ }^{30,38}$ W. Bonivento, ${ }^{15,38}$ S. Borghi,${ }^{54}$ A. Borgia,${ }^{59}$ M. Borsato, ${ }^{7}$ T. J. V. Bowcock, ${ }^{52}$ E. Bowen, ${ }^{40}$ C. Bozzi, ${ }^{16}$ T. Brambach, ${ }^{9}$ J. van den Brand, ${ }^{42}$ J. Bressieux,${ }^{39}$ D. Brett, ${ }^{54}$

M. Britsch ${ }^{10}$ T. Britton, ${ }^{59}$ J. Brodzicka,${ }^{54}$ N. H. Brook ${ }^{46}$ H. Brown,${ }^{52}$ A. Bursche, ${ }^{40}$ G. Busetto, ${ }^{22, e}$ J. Buytaert, ${ }^{38}$ S. Cadeddu, ${ }^{15}$ R. Calabrese, ${ }^{16, b}$ M. Calvi, ${ }^{20, f}$ M. Calvo Gomez, ${ }^{36, g}$ A. Camboni, ${ }^{36}$ P. Campana, ${ }^{18,38}$ D. Campora Perez ${ }^{38}$ A. Carbone, ${ }^{14, \mathrm{~h}}$ G. Carboni, ${ }^{24, \mathrm{i}}$ R. Cardinale, ${ }^{19,38, \mathrm{j}}$ A. Cardini, ${ }^{15}$ H. Carranza-Mejia,${ }^{50}$ L. Carson, ${ }^{50}$ K. Carvalho Akiba, ${ }^{2}$ G. Casse, ${ }^{52}$ L. Cassina, ${ }^{20}$ L. Castillo Garcia, ${ }^{38}$ M. Cattaneo, ${ }^{38}$ Ch. Cauet, ${ }^{9}$ R. Cenci, ${ }^{58}$ M. Charles, ${ }^{8}$ Ph. Charpentier, ${ }^{38}$ S. Chen, ${ }^{54}$ S.-F. Cheung, ${ }^{55}$ N. Chiapolini, ${ }^{40}$ M. Chrzaszcz, ${ }^{40,26}$ K. Ciba ${ }^{38}$ X. Cid Vidal, ${ }^{38}$ G. Ciezarek,${ }^{53}$ P. E. L. Clarke, ${ }^{50}$ M. Clemencic, ${ }^{38}$ H. V. Cliff, ${ }^{47}$ J. Closier, ${ }^{38}$ V. Coco, ${ }^{38}$ J. Cogan, ${ }^{6}$ E. Cogneras, ${ }^{5}$ P. Collins, ${ }^{38}$ A. Comerma-Montells, ${ }^{11}$ A. Contu, ${ }^{15}$ A. Cook,${ }^{46}$ M. Coombes, ${ }^{46}$ S. Coquereau, ${ }^{8}$ G. Corti, ${ }^{38}$ M. Corvo, ${ }^{16, b}$ I. Counts, ${ }^{56}$ B. Couturier,${ }^{38}$ G. A. Cowan, ${ }^{50}$ D. C. Craik, ${ }^{48}$ M. Cruz Torres, ${ }^{60}$ S. Cunliffe, ${ }^{53}$ R. Currie, ${ }^{50}$ C. D'Ambrosio, ${ }^{38}$ J. Dalseno, ${ }^{46}$ P. David, ${ }^{8}$ P. N. Y. David, ${ }^{41}$ A. Davis,${ }^{57}$ K. De Bruyn,${ }^{41}$ S. De Capua,${ }^{54}$ M. De Cian, ${ }^{11}$ J. M. De Miranda, ${ }^{1}$ L. De Paula, ${ }^{2}$ W. De Silva,${ }^{57}$ P. De Simone,${ }^{18}$ D. Decamp, ${ }^{4}$ M. Deckenhoff, ${ }^{9}$ L. Del Buono,${ }^{8}$ N. Déléage, ${ }^{4}$ D. Derkach,${ }^{55}$ O. Deschamps, ${ }^{5}$ F. Dettori,${ }^{38}$ A. Di Canto, ${ }^{38}$ H. Dijkstra, ${ }^{38}$ S. Donleavy, ${ }^{52}$ F. Dordei,${ }^{11}$ M. Dorigo, ${ }^{39}$ A. Dosil Suárez,${ }^{37}$ D. Dossett, ${ }^{48}$ A. Dovbnya ${ }^{43}$ K. Dreimanis ${ }^{52}$ G. Dujany,${ }^{54}$ F. Dupertuis,${ }^{39}$ P. Durante,${ }^{38}$ R. Dzhelyadin, ${ }^{35}$ A. Dziurda ${ }^{26}$ A. Dzyuba ${ }^{30}$ S. Easo, ${ }^{49,38}$ U. Egede,${ }^{53}$ V. Egorychev, ${ }^{31}$ S. Eidelman, ${ }^{34}$ S. Eisenhardt, ${ }^{50}$ U. Eitschberger, ${ }^{9}$ R. Ekelhof, ${ }^{9}$ L. Eklund, ${ }^{51,38}$ I. El Rifai, ${ }^{5}$ Ch. Elsasser,${ }^{40}$ S. Ely, ${ }^{59}$ S. Esen, ${ }^{11}$ H.-M. Evans,${ }^{47}$ T. Evans, ${ }^{55}$ A. Falabella, ${ }^{14}$ C. Färber, ${ }^{11}$ C. Farinelli, ${ }^{41}$ N. Farley, ${ }^{45}$ S. Farry, ${ }^{52}$ RF Fay, ${ }^{52}$ D. Ferguson, ${ }^{50}$ V. Fernandez Albor, ${ }^{37}$ F. Ferreira Rodrigues, ${ }^{1}$ M. Ferro-Luzzi, ${ }^{38}$ S. Filippov, ${ }^{33}$ M. Fiore, ${ }^{16, b}$ M. Fiorini, ${ }^{16, b}$ M. Firlej, ${ }^{27}$ C. Fitzpatrick ${ }^{38}$ T. Fiutowski, ${ }^{27}$ M. Fontana,${ }^{10}$ F. Fontanelli,,${ }^{19}$ R. Forty,${ }^{38}$ O. Francisco, ${ }^{2}$ M. Frank, ${ }^{38}$ C. Frei,${ }^{38}$ M. Frosini, ${ }^{17,38, a}$ J. Fu, ${ }^{21,38}$ E. Furfaro, ${ }^{24, i}$ A. Gallas Torreira, ${ }^{37}$ D. Galli, ${ }^{14, h}$ S. Gallorini, ${ }^{22}$ S. Gambetta, ${ }^{19, j}$ M. Gandelman, ${ }^{2}$ P. Gandini, ${ }^{59}$ Y. Gao, ${ }^{3}$ J. García Pardiñas, ${ }^{37}$ J. Garofoli, ${ }^{59}$ J. Garra Tico, ${ }^{47}$ L. Garrido, ${ }^{36}$ C. Gaspar, ${ }^{38}$ R. Gauld,${ }^{55}$ L. Gavardi, ${ }^{9}$ G. Gavrilov, ${ }^{30}$ E. Gersabeck, ${ }^{11}$ M. Gersabeck, ${ }^{54}$ T. Gershon, ${ }^{48}$ Ph. Ghez ${ }^{4}$ A. Gianelle, ${ }^{22}$ S. Giani, ${ }^{39}$ V. Gibson, ${ }^{47}$

L. Giubega, ${ }^{29}$ V. V. Gligorov, ${ }^{38}$ C. Göbel, ${ }^{60}$ D. Golubkov, ${ }^{31}$ A. Golutvin, ${ }^{53,31,38}$ A. Gomes, ${ }^{1, k}$ H. Gordon, ${ }^{38}$ C. Gotti, ${ }^{20}$ M. Grabalosa Gándara, ${ }^{5}$ R. Graciani Diaz, ${ }^{36}$ L. A. Granado Cardoso, ${ }^{38}$ E. Graugés, ${ }^{36}$ G. Graziani, ${ }^{17}$ A. Grecu, ${ }^{29}$ E. Greening, ${ }^{55}$ S. Gregson, ${ }^{47}$ P. Griffith, ${ }^{45}$ L. Grillo, ${ }^{11}$ O. Grünberg, ${ }^{62}$ B. Gui, ${ }^{59}$ E. Gushchin, ${ }^{33}$ Yu. Guz ${ }^{35,38}$ T. Gys, ${ }^{38}$ C. Hadjivasiliou, ${ }^{59}$ G. Haefeli, ${ }^{39}$ C. Haen, ${ }^{38}$ S. C. Haines, ${ }^{47}$ S. Hall,${ }^{53}$ B. Hamilton, ${ }^{58}$ T. Hampson, ${ }^{46}$ X. Han, ${ }^{11}$

S. Hansmann-Menzemer, ${ }^{11}$ N. Harnew, ${ }^{55}$ S. T. Harnew, ${ }^{46}$ J. Harrison, ${ }^{54}$ J. He,${ }^{38}$ T. Head, ${ }^{38}$ V. Heijne, ${ }^{41}$ K. Hennessy, ${ }^{52}$ P. Henrard, ${ }^{5}$ L. Henry, ${ }^{8}$ J. A. Hernando Morata ${ }^{37}$ E. van Herwijnen, ${ }^{38}$ M. Heß,${ }^{62}$ A. Hicheur, ${ }^{1}$ D. Hill,,${ }^{55}$ M. Hoballah, ${ }^{5}$ C. Hombach ${ }^{54}$ W. Hulsbergen, ${ }^{41}$ P. Hunt, ${ }^{55}$ N. Hussain, ${ }^{55}$ D. Hutchcroft,${ }^{52}$ D. Hynds,${ }^{51}$ M. Idzik,,${ }^{27}$ P. Ilten, ${ }^{56}$

R. Jacobsson, ${ }^{38}$ A. Jaeger, ${ }^{11}$ J. Jalocha,${ }^{55}$ E. Jans, ${ }^{41}$ P. Jaton, ${ }^{39}$ A. Jawahery, ${ }^{58}$ F. Jing, ${ }^{3}$ M. John, ${ }^{55}$ D. Johnson, ${ }^{55}$ C. R. Jones ${ }^{47}$ C. Joram,${ }^{38}$ B. Jost,${ }^{38}$ N. Jurik, ${ }^{59}$ M. Kaballo, ${ }^{9}$ S. Kandybei, ${ }^{43}$ W. Kanso, ${ }^{6}$ M. Karacson, ${ }^{38}$ T. M. Karbach, ${ }^{38}$ S. Karodia, ${ }^{51}$ M. Kelsey, ${ }^{59}$ I. R. Kenyon, ${ }^{45}$ T. Ketel, ${ }^{42}$ B. Khanji, ${ }^{20}$ C. Khurewathanakul, ${ }^{39}$ S. Klaver, ${ }^{54}$ K. Klimaszewski, ${ }^{28}$ O. Kochebina, ${ }^{7}$ M. Kolpin, ${ }^{11}$ I. Komarov, ${ }^{39}$ R. F. Koopman, ${ }^{42}$ P. Koppenburg, ${ }^{41,38}$ M. Korolev, ${ }^{32}$ A. Kozlinskiy, ${ }^{41}$ L. Kravchuk, ${ }^{33}$ K. Kreplin, ${ }^{11}$ M. Kreps, ${ }^{48}$ G. Krocker, ${ }^{11}$ P. Krokovny, ${ }^{34}$ F. Kruse, ${ }^{9}$ W. Kucewicz, ${ }^{26,1}$ M. Kucharczyk, ${ }^{20,26,38, f}$ V. Kudryavtsev, ${ }^{34}$ K. Kurek,${ }^{28}$ T. Kvaratskheliya, ${ }^{31}$ V. N. La Thi,${ }^{39}$ D. Lacarrere, ${ }^{38}$ G. Lafferty, ${ }^{54}$ A. Lai, ${ }^{15}$ D. Lambert,${ }^{50}$ R. W. Lambert, ${ }^{42}$ E. Lanciotti, ${ }^{38}$ G. Lanfranchi, ${ }^{18}$ C. Langenbruch, ${ }^{38}$ B. Langhans, ${ }^{38}$ T. Latham, ${ }^{48}$ C. Lazzeroni, ${ }^{45}$ R. Le Gac, ${ }^{6}$ J. van Leerdam, ${ }^{41}$ J.-P. Lees, ${ }^{4}$ R. Lefèvre, ${ }^{5}$ A. Leflat, ${ }^{32}$ J. Lefrançois, ${ }^{7}$ S. Leo, ${ }^{23}$ O. Leroy, ${ }^{6}$ T. Lesiak, ${ }^{26}$ B. Leverington, ${ }^{11}$ Y. Li, ${ }^{3}$ M. Liles, ${ }^{52}$ R. Lindner, ${ }^{38}$ C. Linn, ${ }^{38}$ F. Lionetto, ${ }^{40}$ B. Liu, ${ }^{15}$ G. Liu, ${ }^{38}$ S. Lohn,${ }^{38}$ I. Longstaff, ${ }^{51}$ J. H. Lopes, ${ }^{2}$ N. Lopez-March, ${ }^{39}$ P. Lowdon, ${ }^{40}$ H. Lu, ${ }^{3}$ D. Lucchesi, ${ }^{22, e}$ H. Luo,${ }^{50}$ A. Lupato, ${ }^{22}$ E. Luppi, ${ }^{16, b}$ O. Lupton, ${ }^{55}$ F. Machefert, ${ }^{7}$ I. V. Machikhiliyan, ${ }^{31}$ F. Maciuc, ${ }^{29}$ O. Maev,${ }^{30}$ S. Malde, ${ }^{55}$ G. Manca, ${ }^{15, m}$ G. Mancinelli, ${ }^{6}$ J. Maratas, ${ }^{5}$ J. F. Marchand, ${ }^{4}$ U. Marconi, ${ }^{14}$ C. Marin Benito, ${ }^{36}$ P. Marino, ${ }^{23, n}$ R. Märki, ${ }^{39}$ J. Marks, ${ }^{11}$ G. Martellotti, ${ }^{25}$ A. Martens, ${ }^{8}$ A. Martín Sánchez, ${ }^{7}$ M. Martinelli, ${ }^{41}$ D. Martinez Santos, ${ }^{42}$ F. Martinez Vidal, ${ }^{64}$ D. Martins Tostes, ${ }^{2}$ A. Massafferri, ${ }^{1}$ R. Matev ${ }^{38}$ Z. Mathe, ${ }^{38}$ C. Matteuzzi, ${ }^{20}$ A. Mazurov, ${ }^{16, b}$ M. McCann,${ }^{53}$ J. McCarthy, ${ }^{45}$ A. McNab, ${ }^{54}$ R. McNulty, ${ }^{12}$ B. McSkelly, ${ }^{52}$ B. Meadows, ${ }^{57}$ F. Meier, ${ }^{9}$ M. Meissner, ${ }^{11}$ M. Merk, ${ }^{41}$ D. A. Milanes, ${ }^{8}$ M.-N. Minard, ${ }^{4}$ N. Moggi, ${ }^{14}$ J. Molina Rodriguez, ${ }^{60}$ S. Monteil, ${ }^{5}$ M. Morandin, ${ }^{22}$ P. Morawski, ${ }^{27}$ A. Mordà, ${ }^{6}$ M. J. Morello, ${ }^{23, n}$ J. Moron, ${ }^{27}$ A.-B. Morris, ${ }^{50}$ R. Mountain, ${ }^{59}$ F. Muheim, ${ }^{50}$ K. Müller, ${ }^{40}$ R. Muresan, ${ }^{29}$ M. Mussini, ${ }^{14}$ B. Muster, ${ }^{39}$ P. Naik,${ }^{46}$ T. Nakada, ${ }^{39}$ 
R. Nandakumar, ${ }^{49}$ I. Nasteva, ${ }^{2}$ M. Needham,${ }^{50}$ N. Neri, ${ }^{21}$ S. Neubert, ${ }^{38}$ N. Neufeld ${ }^{38}$ M. Neuner, ${ }^{11}$ A. D. Nguyen, ${ }^{39}$ T. D. Nguyen ${ }^{39}$ C. Nguyen-Mau, ${ }^{39,0}$ M. Nicol, ${ }^{7}$ V. Niess, ${ }^{5}$ R. Niet, ${ }^{9}$ N. Nikitin, ${ }^{32}$ T. Nikodem, ${ }^{11}$ A. Novoselov, ${ }^{35}$ D. P. O'Hanlon, ${ }^{48}$ A. Oblakowska-Mucha, ${ }^{27}$ V. Obraztsov, ${ }^{35}$ S. Oggero, ${ }^{41}$ S. Ogilvy, ${ }^{51}$ O. Okhrimenko, ${ }^{44}$ R. Oldeman, ${ }^{15, m}$ G. Onderwater, ${ }^{65}$ M. Orlandea, ${ }^{29}$ J. M. Otalora Goicochea, ${ }^{2}$ P. Owen, ${ }^{53}$ A. Oyanguren, ${ }^{64}$ B. K. Pal, ${ }^{59}$ A. Palano, ${ }^{13, p}$ F. Palombo, ${ }^{21, q}$ M. Palutan, ${ }^{18}$ J. Panman, ${ }^{38}$ A. Papanestis,${ }^{49,38}$ M. Pappagallo, ${ }^{51}$ C. Parkes,${ }^{54}$ C. J. Parkinson, ${ }^{9,45}$ G. Passaleva, ${ }^{17}$ G. D. Patel,${ }^{52}$ M. Patel,${ }^{53}$ C. Patrignani, ${ }^{19, j}$ A. Pazos Alvarez,${ }^{37}$ A. Pearce,${ }^{54}$ A. Pellegrino, ${ }^{41}$ M. Pepe Altarelli, ${ }^{38}$ S. Perazzini, ${ }^{14, \mathrm{~h}}$ E. Perez Trigo,${ }^{37}$ P. Perret, ${ }^{5}$ M. Perrin-Terrin, ${ }^{6}$ L. Pescatore,${ }^{45}$ E. Pesen, ${ }^{66}$ K. Petridis,${ }^{53}$ A. Petrolini, ${ }^{19, j}$ E. Picatoste Olloqui ${ }^{36}$ B. Pietrzyk,${ }^{4}$ T. Pilař, ${ }^{48}$ D. Pinci, ${ }^{25}$ A. Pistone, ${ }^{19}$ S. Playfer, ${ }^{50}$ M. Plo Casasus,${ }^{37}$ F. Polci ${ }^{8}$ A. Poluektov, ${ }^{48,34}$ E. Polycarpo, ${ }^{2}$ A. Popov,${ }^{35}$ D. Popov, ${ }^{10}$ B. Popovici, ${ }^{29}$ C. Potterat,${ }^{2}$ E. Price,${ }^{46}$ J. Prisciandaro, ${ }^{39}$ A. Pritchard ${ }^{52}$ C. Prouve, ${ }^{46}$ V. Pugatch,${ }^{44}$ A. Puig Navarro, ${ }^{39}$ G. Punzi, ${ }^{23, r}$ W. Qian, ${ }^{4}$ B. Rachwal, ${ }^{26}$ J. H. Rademacker, ${ }^{46}$ B. Rakotomiaramanana, ${ }^{39}$ M. Rama, ${ }^{18}$ M. S. Rangel, ${ }^{2}$ I. Raniuk, ${ }^{43}$ N. Rauschmayr, ${ }^{38}$ G. Raven,${ }^{42}$ S. Reichert,${ }^{54}$ M. M. Reid, ${ }^{48}$ A. C. dos Reis, ${ }_{1}^{1}$ S. Ricciardi, ${ }^{49}$ S. Richards,${ }^{46}$ M. Rihl,${ }^{38}$ K. Rinnert,${ }^{52}$ V. Rives Molina, ${ }^{36}$ D. A. Roa Romero, P. Robbe, ${ }^{7}$ A. B. Rodrigues, ${ }^{1}$ E. Rodrigues,${ }^{54}$ P. Rodriguez Perez,${ }^{54}$ S. Roiser, ${ }^{38}$ V. Romanovsky, ${ }^{35}$ A. Romero Vidal,${ }^{37}$ M. Rotondo, ${ }^{22}$ J. Rouvinet, ${ }^{39}$ T. Ruf,${ }^{38}$ F. Ruffini, ${ }^{23}$ H. Ruiz, ${ }^{36}$ P. Ruiz Valls, ${ }^{64}$ G. Sabatino, ${ }^{25, i}$ J. J. Saborido Silva, ${ }^{37}$ N. Sagidova, ${ }^{30}$ P. Sail, ${ }^{51}$ B. Saitta, ${ }^{15, m}$ V. Salustino Guimaraes, ${ }^{2}$ C. Sanchez Mayordomo, ${ }^{64}$ B. Sanmartin Sedes, ${ }^{37}$ R. Santacesaria, ${ }^{25}$ C. Santamarina Rios,${ }^{37}$ E. Santovetti, ${ }^{24, i}$ M. Sapunov, ${ }^{6}$ A. Sarti, ${ }^{18, \mathrm{~s}}$ C. Satriano, ${ }^{25, \mathrm{c}}$ A. Satta, ${ }^{24}$ D. M. Saunders, ${ }^{46}$ M. Savrie, ${ }^{16, b}$ D. Savrina,${ }^{31,32}$ M. Schiller,${ }^{42}$ H. Schindler, ${ }^{38}$ M. Schlupp, ${ }^{9}$ M. Schmelling, ${ }^{10}$ B. Schmidt,${ }^{38}$ O. Schneider, ${ }^{39}$ A. Schopper, ${ }^{38}$ M.-H. Schune, ${ }^{7}$ R. Schwemmer ${ }^{38}$ B. Sciascia, ${ }^{18}$ A. Sciubba,${ }^{25}$ M. Seco, ${ }^{37}$ A. Semennikov, ${ }^{31}$ I. Sepp ${ }^{53}$ N. Serra, ${ }^{40}$ J. Serrano, ${ }^{6}$ L. Sestini, ${ }^{22}$ P. Seyfert, ${ }^{11}$ M. Shapkin, ${ }^{35}$ I. Shapoval, ${ }^{16,43, b}$ Y. Shcheglov,${ }^{30}$ T. Shears ${ }^{52}$ L. Shekhtman, ${ }^{34}$ V. Shevchenko, ${ }^{63}$ A. Shires, ${ }^{9}$ R. Silva Coutinho, ${ }^{48}$ G. Simi, ${ }^{22}$ M. Sirendi, ${ }^{47}$ N. Skidmore ${ }^{46}$ T. Skwarnicki, ${ }^{59}$ N. A. Smith, ${ }^{52}$ E. Smith, ${ }^{55,49}$ E. Smith,${ }^{53}$ J. Smith, ${ }^{47}$ M. Smith, ${ }^{54}$ H. Snoek, ${ }^{41}$ M. D. Sokoloff, ${ }^{57}$ F. J. P. Soler, ${ }^{51}$ F. Soomro, ${ }^{39}$ D. Souza, ${ }^{46}$ B. Souza De Paula, ${ }^{2}$ B. Spaan, ${ }^{9}$ A. Sparkes ${ }^{50}$ P. Spradlin, ${ }^{51}$ F. Stagni, ${ }^{38}$ M. Stahl, ${ }^{11}$ S. Stahl,${ }^{11}$ O. Steinkamp,${ }^{40}$ O. Stenyakin, ${ }^{35}$ S. Stevenson, ${ }^{55}$ S. Stoica, ${ }^{29}$ S. Stone,${ }^{59}$ B. Storaci,${ }^{40}$ S. Stracka,${ }^{23,38}$ M. Straticiuc, ${ }^{29}$ U. Straumann, ${ }^{40}$ R. Stroili, ${ }^{22}$ V. K. Subbiah, ${ }^{38}$ L. Sun, ${ }^{57}$ W. Sutcliffe, ${ }^{53}$ K. Swientek, ${ }^{27}$ S. Swientek, ${ }^{9}$ V. Syropoulos, ${ }^{42}$ M. Szczekowski, ${ }^{28}$ P. Szczypka, ${ }^{39,38}$ D. Szilard, ${ }^{2}$ T. Szumlak, ${ }^{27}$ S. T'Jampens, ${ }^{4}$ M. Teklishyn, ${ }^{7}$ G. Tellarini ${ }^{16, b}$ F. Teubert, ${ }^{38}$ C. Thomas, ${ }^{55}$ E. Thomas, ${ }^{38}$ J. van Tilburg, ${ }^{41}$ V. Tisserand, ${ }^{4}$ M. Tobin, ${ }^{39}$ S. Tolk, ${ }^{42}$ L. Tomassetti, ${ }^{16, b}$ S. Topp-Joergensen, ${ }^{55}$ N. Torr, ${ }^{55}$ E. Tournefier, ${ }^{4}$ S. Tourneur, ${ }^{39}$ M. T. Tran, ${ }^{39}$ M. Tresch, ${ }^{40}$ A. Tsaregorodtsev, ${ }^{6}$ P. Tsopelas, ${ }^{41}$ N. Tuning, ${ }^{41}$ M. Ubeda Garcia, ${ }^{38}$ A. Ukleja, ${ }^{28}$ A. Ustyuzhanin, ${ }^{63}$ U. Uwer, ${ }^{11}$ V. Vagnoni, ${ }^{14}$ G. Valenti, ${ }^{14}$ A. Vallier, ${ }^{7}$ R. Vazquez Gomez, ${ }^{18}$

P. Vazquez Regueiro, ${ }^{37}$ C. Vázquez Sierra, ${ }^{37}$ S. Vecchi, ${ }^{16}$ J. J. Velthuis,${ }^{46}$ M. Veltri, ${ }^{17, t}$ G. Veneziano,${ }^{39}$ M. Vesterinen,${ }^{11}$ B. Viaud, ${ }^{7}$ D. Vieira, ${ }^{2}$ M. Vieites Diaz ${ }^{37}$ X. Vilasis-Cardona, ${ }^{36, g}$ A. Vollhardt, ${ }^{40}$ D. Volyanskyy, ${ }^{10}$ D. Voong, ${ }^{46}$

A. Vorobyev,${ }^{30}$ V. Vorobyev,${ }^{34}$ C. Vo $\beta,{ }^{62}$ H. Voss, ${ }^{10}$ J. A. de Vries,${ }^{41}$ R. Waldi,${ }^{62}$ C. Wallace, ${ }^{48}$ R. Wallace, ${ }^{12}$ J. Walsh,${ }^{23}$ S. Wandernoth, ${ }^{11}$ J. Wang, ${ }^{59}$ D. R. Ward, ${ }^{47}$ N. K. Watson, ${ }^{45}$ D. Websdale, ${ }^{53}$ M. Whitehead, ${ }^{48}$ J. Wicht,${ }^{38}$ D. Wiedner, ${ }^{11}$

G. Wilkinson, ${ }^{55}$ M. P. Williams, ${ }^{45}$ M. Williams, ${ }^{56}$ F. F. Wilson, ${ }^{49}$ J. Wimberley,${ }^{58}$ J. Wishahi, ${ }^{9}$ W. Wislicki, ${ }^{28}$ M. Witek, ${ }^{26}$

G. Wormser, ${ }^{7}$ S. A. Wotton, ${ }^{47}$ S. Wright, ${ }^{47}$ S. Wu, ${ }^{3}$ K. Wyllie, ${ }^{38}$ Y. Xie, ${ }^{61}$ Z. Xing, ${ }^{59}$ Z. Xu, ${ }^{39}$ Z. Yang, ${ }^{3}$ X. Yuan, ${ }^{3}$ O. Yushchenko, ${ }^{35}$ M. Zangoli, ${ }^{14}$ M. Zavertyaev, ${ }^{10, u}$ L. Zhang, ${ }^{59}$ W. C. Zhang, ${ }^{12}$ Y. Zhang, ${ }^{3}$ A. Zhelezov, ${ }^{11}$ A. Zhokhov, ${ }^{31}$ L. Zhong ${ }^{3}$ and A. Zvyagin ${ }^{38}$

(LHCb Collaboration)

${ }^{1}$ Centro Brasileiro de Pesquisas Físicas (CBPF), Rio de Janeiro, Brazil

${ }^{2}$ Universidade Federal do Rio de Janeiro (UFRJ), Rio de Janeiro, Brazil

${ }^{3}$ Center for High Energy Physics, Tsinghua University, Beijing, China

${ }^{4} L A P P$, Université de Savoie, CNRS/IN2P3, Annecy-Le-Vieux, France

${ }^{5}$ Clermont Université, Université Blaise Pascal, CNRS/IN2P3, LPC, Clermont-Ferrand, France ${ }^{6} \mathrm{CPPM}$, Aix-Marseille Université, CNRS/IN2P3, Marseille, France

${ }^{7} L A L$, Université Paris-Sud, CNRS/IN2P3, Orsay, France

${ }^{8}$ LPNHE, Université Pierre et Marie Curie, Université Paris Diderot, CNRS/IN2P3, Paris, France

${ }^{9}$ Fakultät Physik, Technische Universität Dortmund, Dortmund, Germany

${ }^{10}$ Max-Planck-Institut für Kernphysik (MPIK), Heidelberg, Germany

${ }^{11}$ Physikalisches Institut, Ruprecht-Karls-Universität Heidelberg, Heidelberg, Germany

${ }^{12}$ School of Physics, University College Dublin, Dublin, Ireland

${ }^{13}$ Sezione INFN di Bari, Bari, Italy 


\author{
${ }^{14}$ Sezione INFN di Bologna, Bologna, Italy \\ ${ }^{15}$ Sezione INFN di Cagliari, Cagliari, Italy \\ ${ }^{16}$ Sezione INFN di Ferrara, Ferrara, Italy \\ ${ }^{17}$ Sezione INFN di Firenze, Firenze, Italy \\ ${ }^{18}$ Laboratori Nazionali dell'INFN di Frascati, Frascati, Italy \\ ${ }^{19}$ Sezione INFN di Genova, Genova, Italy \\ ${ }^{20}$ Sezione INFN di Milano Bicocca, Milano, Italy \\ ${ }^{21}$ Sezione INFN di Milano, Milano, Italy \\ ${ }^{22}$ Sezione INFN di Padova, Padova, Italy \\ ${ }^{23}$ Sezione INFN di Pisa, Pisa, Italy \\ ${ }^{24}$ Sezione INFN di Roma Tor Vergata, Roma, Italy \\ ${ }^{25}$ Sezione INFN di Roma La Sapienza, Roma, Italy \\ ${ }^{26}$ Henryk Niewodniczanski Institute of Nuclear Physics Polish Academy of Sciences, Kraków, Poland \\ ${ }^{27}$ AGH-University of Science and Technology, Faculty of Physics and Applied Computer Science, Kraków, Poland \\ ${ }^{28}$ National Center for Nuclear Research (NCBJ), Warsaw, Poland \\ ${ }^{29}$ Horia Hulubei National Institute of Physics and Nuclear Engineering, Bucharest-Magurele, Romania \\ ${ }^{30}$ Petersburg Nuclear Physics Institute (PNPI), Gatchina, Russia \\ ${ }^{31}$ Institute of Theoretical and Experimental Physics (ITEP), Moscow, Russia \\ ${ }^{32}$ Institute of Nuclear Physics, Moscow State University (SINP MSU), Moscow, Russia \\ ${ }^{33}$ Institute for Nuclear Research of the Russian Academy of Sciences (INR RAN), Moscow, Russia \\ ${ }^{34}$ Budker Institute of Nuclear Physics (SB RAS) and Novosibirsk State University, Novosibirsk, Russia \\ ${ }^{35}$ Institute for High Energy Physics (IHEP), Protvino, Russia \\ ${ }^{36}$ Universitat de Barcelona, Barcelona, Spain \\ ${ }^{37}$ Universidad de Santiago de Compostela, Santiago de Compostela, Spain \\ ${ }^{38}$ European Organization for Nuclear Research (CERN), Geneva, Switzerland \\ ${ }^{39}$ Ecole Polytechnique Fédérale de Lausanne (EPFL), Lausanne, Switzerland \\ ${ }^{40}$ Physik-Institut, Universität Zürich, Zürich, Switzerland \\ ${ }^{41}$ Nikhef National Institute for Subatomic Physics, Amsterdam, The Netherlands \\ ${ }^{42}$ Nikhef National Institute for Subatomic Physics and VU University Amsterdam, Amsterdam, The Netherlands \\ ${ }^{43}$ NSC Kharkiv Institute of Physics and Technology (NSC KIPT), Kharkiv, Ukraine \\ ${ }^{44}$ Institute for Nuclear Research of the National Academy of Sciences (KINR), Kyiv, Ukraine \\ ${ }^{45}$ University of Birmingham, Birmingham, United Kingdom \\ ${ }^{46}$ H. H. Wills Physics Laboratory, University of Bristol, Bristol, United Kingdom \\ ${ }^{47}$ Cavendish Laboratory, University of Cambridge, Cambridge, United Kingdom \\ ${ }^{48}$ Department of Physics, University of Warwick, Coventry, United Kingdom \\ ${ }^{49}$ STFC Rutherford Appleton Laboratory, Didcot, United Kingdom \\ ${ }^{50}$ School of Physics and Astronomy, University of Edinburgh, Edinburgh, United Kingdom \\ ${ }^{51}$ School of Physics and Astronomy, University of Glasgow, Glasgow, United Kingdom \\ ${ }^{52}$ Oliver Lodge Laboratory, University of Liverpool, Liverpool, United Kingdom \\ ${ }^{53}$ Imperial College London, London, United Kingdom \\ ${ }^{54}$ School of Physics and Astronomy, University of Manchester, Manchester, United Kingdom \\ ${ }^{55}$ Department of Physics, University of Oxford, Oxford, United Kingdom \\ ${ }^{56}$ Massachusetts Institute of Technology, Cambridge, Massachusetts, USA \\ ${ }^{57}$ University of Cincinnati, Cincinnati, Ohio, USA \\ ${ }^{58}$ University of Maryland, College Park, Maryland, USA \\ ${ }^{59}$ Syracuse University, Syracuse, New York, USA \\ ${ }^{60}$ Pontifícia Universidade Católica do Rio de Janeiro (PUC-Rio), Rio de Janeiro, \\ Brazil (associated with Universidade Federal do Rio de Janeiro (UFRJ), Rio de Janeiro, Brazil) \\ ${ }^{61}$ Institute of Particle Physics, Central China Normal University, Wuhan, Hubei, \\ China (associated with Center for High Energy Physics, Tsinghua University, Beijing, China) \\ ${ }^{62}$ Institut für Physik, Universität Rostock, Rostock, Germany (associated with Physikalisches Institut, \\ Ruprecht-Karls-Universität Heidelberg, Heidelberg, Germany) \\ ${ }^{63}$ National Research Centre Kurchatov Institute, Moscow, Russia (associated with Institute of Theoretical \\ and Experimental Physics (ITEP), Moscow, Russia) \\ ${ }^{64}$ Instituto de Fisica Corpuscular (IFIC), Universitat de Valencia-CSIC, Valencia, \\ Spain (associated with Universitat de Barcelona, Barcelona, Spain) \\ ${ }^{65}$ KVI-University of Groningen, Groningen, The Netherlands \\ (associated with Nikhef National Institute for Subatomic Physics, Amsterdam, The Netherlands)
}


${ }^{66}$ Celal Bayar University, Manisa, Turkey

(associated with European Organization for Nuclear Research (CERN), Geneva, Switzerland)

${ }^{a}$ Also at Università di Firenze, Firenze, Italy.

${ }^{\mathrm{b}}$ Also at Università di Ferrara, Ferrara, Italy.

${ }^{\mathrm{c}}$ Also at Università della Basilicata, Potenza, Italy.

${ }^{\mathrm{d}}$ Also at Università di Modena e Reggio Emilia, Modena, Italy.

${ }^{\mathrm{e}}$ Also at Università di Padova, Padova, Italy.

${ }^{\mathrm{f}}$ Also at Università di Milano Bicocca, Milano, Italy.

${ }^{g}$ Also at LIFAELS, La Salle, Universitat Ramon Llull, Barcelona, Spain.

${ }^{\mathrm{h}}$ Also at Università di Bologna, Bologna, Italy.

${ }^{i}$ Also at Università di Roma Tor Vergata, Roma, Italy.

${ }^{\mathrm{j}}$ Also at Università di Genova, Genova, Italy.

${ }^{\mathrm{k}}$ Also at Universidade Federal do Triângulo Mineiro (UFTM), Uberaba-MG, Brazil.

${ }^{1}$ Also at AGH - University of Science and Technology, Faculty of Computer Science, Electronics and Telecommunications, Kraków, Poland.

${ }^{\mathrm{m}}$ Also at Università di Cagliari, Cagliari, Italy.

${ }^{\mathrm{n}}$ Also at Scuola Normale Superiore, Pisa, Italy.

${ }^{\circ}$ Also at Hanoi University of Science, Hanoi, Vietnam.

${ }^{\mathrm{p}}$ Also at Università di Bari, Bari, Italy.

${ }^{\mathrm{q}}$ Also at Università degli Studi di Milano, Milano, Italy.

${ }^{\mathrm{r}}$ Also at Università di Pisa, Pisa, Italy.

${ }^{\mathrm{s}}$ Also at Università di Roma La Sapienza, Roma, Italy.

${ }^{t}$ Also at Università di Urbino, Urbino, Italy.

"Also at P. N. Lebedev Physical Institute, Russian Academy of Science (LPI RAS), Moscow, Russia. 